\title{
Attraction between weakly charged silica spheres at a water-air interface induced by surface-charge heterogeneity
}

\author{
Wei Chen, ${ }^{1}$ Susheng Tan, ${ }^{2}$ Yi Zhou, ${ }^{1}$ Tai-Kai Ng, ${ }^{1}$ Warren T. Ford, ${ }^{2}$ and Penger Tong ${ }^{1, *}$ \\ ${ }^{1}$ Department of Physics, Hong Kong University of Science and Technology, Clear Water Bay, Kowloon, Hong Kong, China \\ ${ }^{2}$ Department of Chemistry, Oklahoma State University, Stillwater, Oklahoma 74078, USA
}

(Received 1 January 2009; published 14 April 2009)

\begin{abstract}
We report an optical and atomic force microscopic (AFM) study of interactions between weakly charged silica spheres at a water-air interface. Attractive interactions are observed at intermediate interparticle distances and the amplitude of the attraction increases with the amount of salt $(\mathrm{NaCl})$ added into the water phase. AFM images obtained in the salty water show the formation of patchy charge domains of size $\sim 100 \mathrm{~nm}$ on the silica surface. The experiment suggests that surface heterogeneity produced during ionization plays an important role in the generation of attractions between like-charged particles at the interface.
\end{abstract}

DOI: 10.1103/PhysRevE.79.041403

PACS number(s): 82.70.Dd, 41.20.Cv, 61.20.-p, 68.05.Gh

\section{INTRODUCTION}

Screened Coulomb repulsion between molecules of same charge is a fundamental force necessary for the stability and structure formation of various macromolecules in aqueous solutions $[1,2]$. For two identical charged spheres of diameter $d$ and effective charge $q$ in an aqueous solution of dielectric constant $\epsilon_{0} \epsilon$, their electrostatic interaction potential has the form $[1,2]$

$$
U_{C}(r)=\frac{q^{2} \exp \left[-(r-d) / \lambda_{D}\right]}{4 \pi \epsilon_{0} \epsilon\left[1+d /\left(2 \lambda_{D}\right)\right]^{2} r}
$$

where $r$ is the sphere's center-to-center separation and $\lambda_{D}$ is the Debye screening length. This screened Coulomb repulsion together with short-ranged van der Waals attraction are widely used to calculate the stability and phase behavior of charged colloidal systems [1-4].

In recent years, however, there has been an increasing number of experiments reporting attractive interactions rather than screened Coulomb repulsion between charged colloidal particles near a liquid-solid interface [5-8] or at a liquid-liquid interface [9-12]. Like-charge attractions were also found in the (bulk) salt solutions of cytoskeletal filamentous actin [13] and DNA [14]. Because of its fundamental interest and important implications in colloid science and biology, the vexing issue of like-charge attractions has been under intensive theoretical and experimental scrutinies for many years $[3,4,11,15,16]$. Understanding of such attraction is extremely important as it determines the stability and structure formation of a variety of macromolecules in aqueous solutions, including proteins and DNA $[1,2,13,14]$. Despite the intensive theoretical and experimental investigations over the past decade, a satisfying explanation for the origin of the attraction still remains illusive. The lack of progress is partially due to a lack of well-controlled experimental studies of particle interactions at the interface. Most early experiments only reported the formation of colloidal clusters and no quantitative measurement of the interaction

\footnotetext{
*Corresponding author; penger@ust.hk
}

parameters was provided $[5,9,10]$. There were few experimental studies reported the measurement of the interaction potential but they were conducted without varying any control parameter $[6-8,11,17]$. The lack of experimental control parameters makes it difficult to distinguish possible experimental artifacts $[15,16]$ from the measured attraction and to have a quantitative comparison with different theoretical models.

While there is no generally accepted explanation, counterions are thought to play an essential roll in determining the colloidal interactions. The theoretical calculations assume idealized boundary conditions, such as a uniform charge distribution, whereas the colloidal systems used in the experiment often reveal interesting but unexpected deviations. Recently, it was found [18-21] that the intrinsic surface charge distribution of polystyrene latex spheres, which are the representative of charged particles commonly used, is not uniform as is commonly believed. An important question is whether such surface heterogeneity is a common cause for the like-charge attractions observed in other colloidal systems, such as silica spheres which represent another class of charged particles [5-8].

In this paper, we report results of a systematic experimental study of interactions between charged silica spheres at a water-air interface. Attractive interactions are observed at intermediate interparticle distances and the amplitude of the attraction is found to increase with the amount of salt $(\mathrm{NaCl})$ added into the water phase. The well-controlled measurements with the salt concentration effectively eliminate the possibility of experimental artifacts. The experiment shows that the ionization of the smooth silica surface takes place nonuniformly, and the effect of the nonuniform ionization is further enhanced when a salt is added into the water. With the measured interaction parameters and their dependence on the salt concentration, we are able to carry out a quantitative comparison among different theoretical models.

The remainder of the paper is organized as follows. We first describe the apparatus and the experimental method in Sec. II. Experimental results and discussions are presented in Sec. III. Finally, the work is summarized in Sec. IV. 


\section{EXPERIMENT}

\section{A. Apparatus and sample preparation}

We follow the same procedures as described in Ref. [19] for the experimental setup and the sample preparation and cleaning. The water-air interface is prepared using a homemade Teflon trough of rectangular shape with width $10 \mathrm{~cm}$, length $28 \mathrm{~cm}$, and height $1.0 \mathrm{~cm}$. An optical window that allows observation of the interfacial particles from below is installed at the center of the bottom surface of the trough. The window holder consists of a stainless steel insert, which raises the optical window to within $2 \mathrm{~mm}$ of the water surface. The top surface of the insert is of a cone shape with a central hole of diameter $1.3 \mathrm{~cm}$. The bottom of the hole is sealed with a 0.1 -mm-thick glass cover slip. The sidewall of the hole together with the bottom glass slip forms a sample cell which has an effective height of $1.0 \mathrm{~mm}$. Care is taken to suppress unwanted surface flow at the interface. The sharp top edge of the stainless steel insert provides a strong pining circle for the interface. This nonslip boundary condition together with the small surface area effectively suppress the surface flow. In addition, a glass cover is used to shelter the interface from air flows in the surrounding. The entire trough is placed on the sample stage of an inverted microscope (Leica DM-IRB) so that the motion of the interfacial particles can be viewed from below through an objective with magnification varied from $40 \times$ to $94.5 \times$. Higher magnifications are used for samples with higher particle concentrations.

The silica spheres used in the experiment were purchased from Duke Scientific. The diameter of these particles is $d$ $=0.73 \pm 0.04 \mu \mathrm{m}$ and they have anionic $\mathrm{SiO}^{-}$groups on the surface when dispersed in water. In the experiment to be discussed below, we find the surface charge density of the silica spheres is $\sigma \simeq 0.01 \mu \mathrm{C} / \mathrm{cm}^{2}$, which is $\sim 200$ times smaller than that of polystyrene latex spheres. Polystyrene and silica spheres are the representative of the charged particles commonly used in colloidal science. Unlike the polystyrene spheres, however, the silica spheres appear to have a macroscopically uniform surface (see below). The purchased aqueous samples are surfactant free, but we find that chemical impurities in the solution can still cause substantial increase in the measured surface pressure isotherm. Surface pressure measurements of the supernatant solution (without particles) are carried out using a commercial LangmuirBlodgett trough (Type-611, Nima).

Great care is taken to clean the particle samples and the water-air interface. The received aqueous samples are thoroughly cleaned with methanol via repeated centrifugation for 6-7 times until the final supernatant is tested to be clean. Surface pressure measurements reveal that no detectable impurity is found in the cleaned particle samples. A cleaning procedure [22] is followed to clean the homemade trough and the water-air interface. The particle-methanol solution is then injected onto a clean water-air interface using a syringe pump with a $1 \mathrm{ml}$ glass syringe holding the particle-methanol solution. Methanol drops wet the water surface quickly and the evaporation of the thin methanol layer only takes a few seconds. After the evaporation of methanol, a well-dispersed monolayer of particles forms on the interface. By following these experimental procedures, we are able to remove impurities from the interface and routinely produce a wellbehaved and nicely dispersed monolayer of silica particles at the water-air interface. Individual particles at the interface undergo vigorous Brownian motion and remain stable with various concentrations for days. Such a two-dimensional colloidal system is an ideal system for the study attempted here. The particle's spatial configuration and dynamics are clearly visible without multiple scattering and are not influenced by a nearby wall.

\section{B. Optical microscopy}

The equilibrium configurations of the interfacial particles are viewed with the Leica inverted microscope. Both the phase contrast and bright field microscopies are used to obtain good images of the particles undergoing Brownian motion. For high-concentration samples, we find that the images obtained with the phase contrast appear to have slightly better contrast. For dilute samples, both the phase contrast and bright field work well. Usually, we use the phase contrast to obtain the particle images. Image sequences are recorded by a digital camera (CoolSNAP-cf, Photometrics) and then stored into a host computer. The spatial resolution of each image is set at $1392 \times 1040$ pixels with a 12-bit dynamic range for the gray level. The cross-section of each particle occupies $\sim 50$ pixels in the image. The frame rate is set at 1 frame/s and the exposure time is $30 \mathrm{~ms}$.

Commercial image software (IMAGEPRO, MediaCybernetics) is used for camera control and the determination of particle positions in each image with a spatial resolution of 60 $100 \mathrm{~nm}$. From the obtained particle positions, one can calculate the pair-correlation function $g(r)$ as follows. First, for a test particle $i$, we count the number $n_{i}(r)$ of particles in a circular bin of radius $r$ and width $d r$ centered on the test particle. According to the definition of $g(r)$, one has $g(r)$ $=n_{i}(r) /[\rho(2 \pi r d r)]$, where $2 \pi r d r$ is the bin area and $\rho$ $=N / A$ is the number density of the particles in an image (with $N$ being the total number of particles in the image and $A$ being the area of the image). To have better statistics, we then sum over all such circular bins in the image and obtain [23]

$$
g(r)=\frac{\sum_{i} n_{i}(r)}{\sum_{i} \rho(2 \pi r d r)}=\frac{2 N(r)}{A \rho^{2}(2 \pi r d r)},
$$

where $N(r)$ is the number of particle pairs at separation $r$ in each image. In obtaining Eq. (2), we have assumed that all the circular bins remain inside the image. To include those circular bins, which only partially remain inside the image (the edge effect), one needs to subtract out the number of the particles occupied in the total bin area, $\Sigma_{i} \delta A_{i}^{\text {>edge }}(r)$, outside the image (overcounted bin area) from the denominator. Therefore, we have 


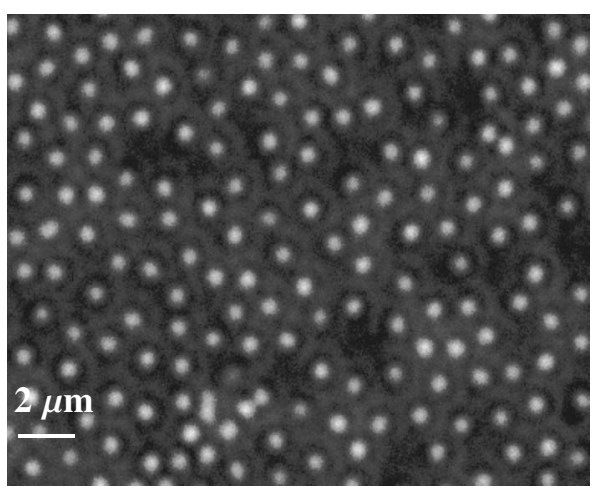

FIG. 1. Equilibrium configuration of silica spheres at the waterair interface with area fraction $n \simeq 0.11$. The interface is prepared using fresh deionized water.

$$
\begin{aligned}
g(r) & =\frac{2 N(r)}{\sum_{i} \rho\left[2 \pi r d r-\delta A_{i}^{>\text {edge }}(r)\right]} \\
& =\frac{2 N(r)}{A \rho^{2} 2 \pi r d r-\rho \sum_{i} \delta A_{i}^{>\text {edge }}(r)},
\end{aligned}
$$

where $\delta A_{i}^{\text {>edge }}(r)$ is the area of the circular bin of the $i$ th particle remained outside the image. Typically, we use 1000 images, each containing $\sim 100$ particles, to calculate $g(r)$. This corresponds to an average over $10^{5}$ particles, ensuring that the statistical averaging is adequate.

\section{Atomic force microscopy}

Atomic force microscopy (AFM) measurements are conducted using a multimode scanning probe microscope equipped with a NanoScope IIIa controller (Digital Instruments, Veeco Metrology Group) and operated under the tapping mode. Commercial silicon microcantilever probes (MikroMasch) with tip radius 5-10 nm and spring constant 2-5 N/m are used for AFM imaging. A monolayer sample of silica spheres for AFM imaging is obtained by depositing a drop of a dilute aqueous suspension of particles on a newly cleaved mica substrate and drying at room temperature. A fluid cell is used to image the silica spheres and oxidized silicon wafers under water. The sample to be imaged is first transferred to the cell substrate, and then deionized water (purified with a Barnstead three column e-pure system to a resistivity of $18 \mathrm{M} \Omega \mathrm{cm}$ ) or $\mathrm{NaCl}$ aqueous solution is introduced to the fluid cell from a reservoir. Topographic and phase images are obtained simultaneously at a driving frequency of $120 \mathrm{kHz}$ for the probe oscillation and the scanning rate of the images is set at $1 \mathrm{~Hz}$. The AFM measurements are performed in the attractive regime with a fixed set-point ratio, $A_{\mathrm{sp}} / A_{0}=0.9$, where $A_{0}$ is the free oscillation amplitude and $A_{\mathrm{sp}}$ is a constant amplitude maintained during imaging.

\section{EXPERIMENTAL RESULTS AND DISCUSSIONS}

\section{A. Measurements of the pair potential}

Figure 1 shows an optical image of silica spheres at the water-air interface. Under phase contrast microscopy, the
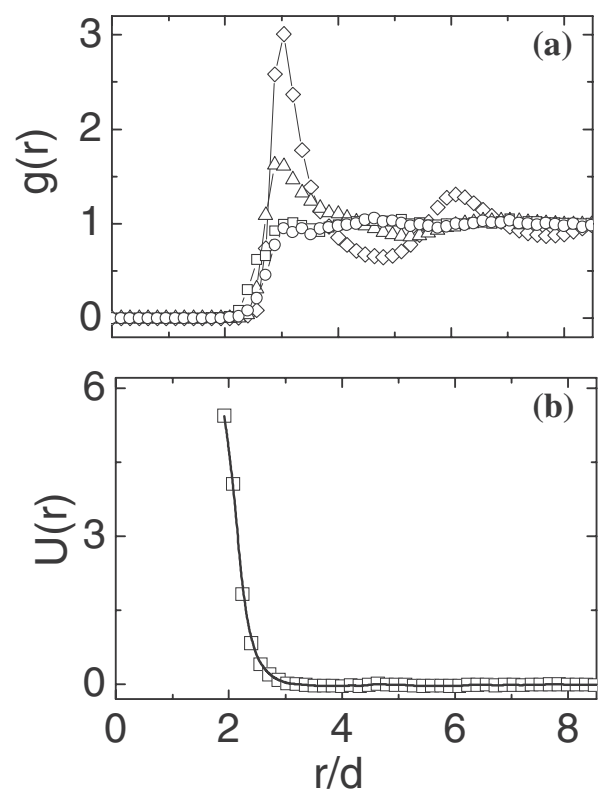

FIG. 2. (a) Measured pair-correlation function $g(r)$ as a function of $r / d$ for four area fractions: $n=0.012$ (squares), $n=0.019$ (circles), $n=0.1$ (triangles), and $n=0.16$ (diamonds). Fresh deionized water is used in the experiment. The solid lines are drawn to guide the eyes. (b) Interaction potential $U(r) / k_{B} T$ vs $r / d$ converted from the measured $g(r)$ at $n=0.012$. The solid curve shows a least-squared fit to Eq. (1).

particles appear as clear circles with uniform size distribution. These particles remain in focus under high magnification, indicating that the silica spheres are closely bound to the interface and their vertical position is determined by an energy minimum, much larger than $k_{B} T$, that keeps them at the interface [24]. The characteristic surface energy for the interfacial particles of radius $a$ scales as [24] $\pi a^{2} \gamma$, which is $10^{7} k_{B} T$ for a water-air interface of interfacial tension $\gamma$ $\simeq 72 \mathrm{mN} / \mathrm{m}$. The surface energy remains at $10^{2} k_{B} T$ even when the particle's contact area at the interface is reduced to $10 \mathrm{~nm}^{2}$. This value is still very large compared with the gravitational energy $\Delta m g a \simeq 0.4 k_{B} T$ for a silica sphere of buoyant mass $\Delta m$ under earth's gravity $g$. Using an estimated contact angle of $60^{\circ}$ [25], we find approximately $3 / 4$ of the particle (by diameter) is immersed in water. Because the gravitational energy of the particles is much smaller than the relevant energy of the interface, the particles can always choose an equilibrium (vertical) position without introducing long-range deformations to the interface. Therefore, the usual capillary effect is not applicable to micron-sized spheres.

Figure 2(a) shows the measured pair-correlation function $g(r)$ for four different area fractions. The area fraction $n$ occupied by the interfacial particles is defined as $n$ $=\pi a^{2} N / A$, where $N$ is the total number of particles in the area $A$. In the plot, the interparticle distance $r$ is scaled by the particle diameter $d$. The measured $g(r)$ does not change much at low area fractions $(n \lesssim 0.02)$ and shows a short repulsive range $0-2.5 d$, in which $g(r) \simeq 0$. At higher area fractions, the measured $g(r)$ starts to oscillate with a dominant peak at $r \simeq 3 d$. The new length scale reflects a partial order- 


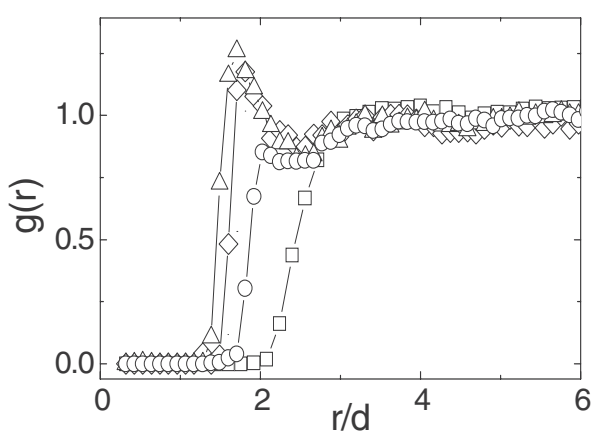

FIG. 3. Measured $g(r)$ as a function of $r / d$ at $n \simeq 0.01$ with different $\mathrm{NaCl}$ concentrations: fresh deionized water (squares), $5 \mu \mathrm{M}$ (circles), $20 \mu \mathrm{M}$ (diamonds), and $50 \mu \mathrm{M}$ (triangles). The solid lines are drawn to guide the eyes.

ing of the particles at the interface with a preferred interparticle separation.

When the surface coverage is low, the measured $g(r)$ is related to the interaction potential $U(r)$ through the Boltzmann factor, $g(r) \simeq \exp \left[-U(r) / k_{B} T\right]$, where $k_{B} T$ is the thermal energy of individual particles. To avoid the crowding effect at finite particle concentrations, we calculate the many-body corrections to $U(r)$ using the hypernetted chain and Percus-Yevick approximations [23]. It is found that these corrections are very small when the area fraction is in the range $n \leqq 0.018$ and do not affect the results to be discussed below. Figure 2(b) shows the resulting $U(r) / k_{B} T$, which can be well fitted to the screened Coulomb potential shown in Eq. (1) with $q=(2000 \pm 550) e$ and $\lambda_{D} \simeq 200 \pm 20 \mathrm{~nm}$ (solid line). The fitting results are consistent with those obtained previously for silica spheres fully immersed in water [26]. Assuming that the charges are uniformly distributed on the entire surface of the silica sphere, the fitted value of $q$ gives an effective surface charge density $\sigma \simeq 0.01 \mu \mathrm{C} / \mathrm{cm}^{2}$.

The measured $g(r)$ is found to change sensitively with the amount of salt $(\mathrm{NaCl})$ added in the water phase. In the concentration range $0-200 \mu \mathrm{M}$, we measure $g(r)$ for 12 different values of the salt concentration $C$. For $C>200 \mu \mathrm{M}$, the silica spheres become unstable and start to form aggregates at the interface (due to van der Waals attraction). Figure 3 compares the pair-correlation function $g(r)$ measured at $n$ $\simeq 0.01$ in fresh deionized water (squares) with those obtained at the same area fraction but having different $\mathrm{NaCl}$ concentrations: $5 \mu \mathrm{M}$ (circles), $20 \mu \mathrm{M}$ (diamonds), and $50 \mu \mathrm{M}$ (triangles). The measured $g(r)$ is so sensitive to the trace amount of salt in water that we find it slowly changes with time. Slow dissolution of ions into the aqueous phase from atmospheric $\mathrm{CO}_{2}$ changes the value of the Debye screening length $\lambda_{D}$. The measured $g(r)$ in 1-2 day old water sample is found to be equivalent to those measured in the $10-15 \mu \mathrm{M}$ $\mathrm{NaCl}$ solution.

Figure 4(a) shows the resulting $U(r) / k_{B} T$ for the four sample salt concentrations. By adding merely $50 \mu \mathrm{M} \mathrm{NaCl}$, the repulsive range of the measured $U(r)$ is shortened by a distance close to the particle diameter $d$. Such a reduction in the screening range opens up a window of length scales, in which one could observe weaker interactions. Indeed, Fig. 4(a) shows a gradual development of a repulsive barrier of

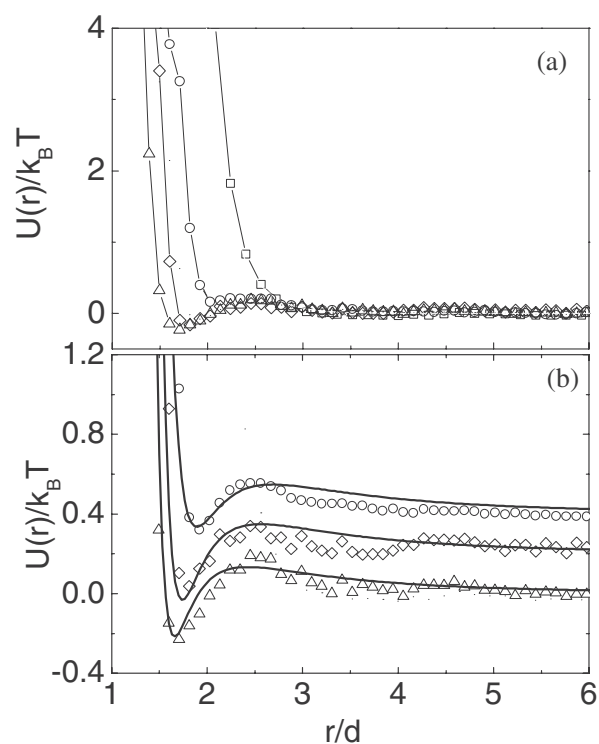

FIG. 4. (a) Interaction potential $U(r) / k_{B} T$ as a function of $r / d$ obtained at $n \simeq 0.01$ with different $\mathrm{NaCl}$ concentrations: fresh deionized water (squares), $5 \mu \mathrm{M}$ (circles), $20 \mu \mathrm{M}$ (diamonds), and $50 \mu \mathrm{M}$ (triangles). These curves are extracted from the measured $g(r)$ shown in Fig. 3. The solid lines are drawn to guide the eyes. (b) Enlarged plots of $U(r) / k_{B} T$ at different salt concentrations: $10 \mu \mathrm{M}$ (circles), $20 \mu \mathrm{M}$ (diamonds), and $50 \mu \mathrm{M}$ (triangles). For clarity, the origin of the vertical axis for the top two curves are shifted by an amount of 0.4 and 0.2 , respectively. The solid curves show the fits to Eq. (4).

$\sim 0.15 k_{B} T$ in amplitude at $r_{1} \simeq 2.5 d$ and an attractive well of approximately $-0.3 k_{B} T$ in amplitude at $r_{0} \simeq 1.7 d$. The magnified plots in Fig. 4(b) reveal the changes in $U(r)$ more clearly. Evidently both the repulsive barrier and the attractive well are located outside the screening range $\lambda_{D}$. Similar behavior is also observed when a divalent salt $\left(\mathrm{CaCl}_{2}\right)$ is added to the aqueous phase.

The measured $U(r)$ for different salt concentrations is well described by the form

$$
\frac{U(r)}{k_{B} T}=\frac{U_{C}(r)}{k_{B} T}-A_{1}\left(\frac{d}{r}\right)^{6}+A_{2}\left(\frac{d}{r}\right)^{3},
$$

where the last two terms with $A_{1}$ and $A_{2}$ as the fitting parameters result from the electrostatic potential in the air. These two terms are unscreened because no ion is in the air $[24,27,28]$. The first term $U_{C}(r)$ given by Eq. (1) is the leading-order potential in the water and has two parameters, $\lambda_{D}$ and $q$; both are functions of the salt concentration $C$. The fittings shown in Fig. 4(b) (solid lines) give $\left(\lambda_{D}, q\right)$ $=(96 \pm 10 \mathrm{~nm}, 3300 \pm 850 e),(71 \pm 8 \mathrm{~nm}, 7000 \pm 2000 e)$, and $(59 \pm 8 \mathrm{~nm}, 8800 \pm 2500 e)$, respectively, with increasing $C$. The fitted values of $\lambda_{D}$ agree with the theory $\left(\lambda_{D}\right.$ $=304 / C^{1 / 2} \mathrm{~nm}$, where $C$ is in units of $\mu \mathrm{M}$ [1]), except for the last number, which has relative larger uncertainties because of our resolution limit in resolving small interparticle separations. The increase in the fitted value of $q$ with $C$ agrees with the mean-field calculation for the ionization of silica spheres [26]. 
The $1 / r^{3}$ repulsion in Eq. (4) results from the interaction between the induced out-of-plane dipoles at the interface $[24,29]$. The surface charges on the particle and the asymmetric counterion clouds at an average separation $\lambda_{D}$ from the sphere's surface immersed in water form a dipole moment, which points downward perpendicular to the interface and has a magnitude $[29] \widetilde{P}_{z} \simeq q \lambda_{D} / \sqrt{\epsilon}$, where $\epsilon(\simeq 80)$ is the dielectric constant of water. With the measured values of the screening length and charge $\left(\lambda_{D}, q\right)=(96 \mathrm{~nm}, 3300 e)$, we calculate the effective dipole moment $\widetilde{P}_{z}$ and find [29] $A_{2}$ $\simeq 2 \widetilde{P}_{z}^{2} /\left(4 \pi \epsilon_{0} \epsilon d^{3} k_{B} T\right) \simeq 4.4$. The fittings shown in Fig. 4(b) give $A_{2}=5.8 \pm 0.5,5.2 \pm 0.5$, and $3.8 \pm 0.5$, respectively, with increasing $C$, which are close to the expected value.

The $1 / r^{6}$ attraction in Eq. (4) is a new finding of the experiment. The fittings shown in Fig. 4 (b) give $A_{1} \simeq 57,42$, and 27 , respectively, with increasing $C$. The balance of this attraction with $U_{C}(r)$ gives rise to the attractive well at $r_{0}$ and that with the $1 / r^{3}$ dipole repulsion produces the repulsive barrier at $r_{1}$. There are several candidates for the origin of the attraction with a $1 / r^{6}$ dependence at the large $r / d$ limit. They include the van der Waals attraction [1], the electrostatic capillary attraction $[11,12,30-32]$, and a van-der-Waals-type attraction due to correlated fluctuations of counterions between two nearby particles [33]. In Sec. III B, we show that the amplitude of these attractive forces is simply too small (at least $10^{-2}$ times smaller) to account for the fitted values of $A_{1}$.

\section{B. Fluctuation-induced attractions}

It is known that correlated fluctuations of charges can cause attractive interactions between two charged particles $[1,2,14]$. There are several candidates for such fluctuationinduced attractions. The van der Waals attraction has the form $[1,2]$

$$
U_{V}(r) \simeq-\frac{H}{36}\left(\frac{d}{r}\right)^{6},
$$

at the large $r / d$ limit, where $H$ is the Hamaker constant. For fused silica in vacuum with $H \simeq 6.6 \times 10^{-20} \mathrm{~J}$ (the value of $H$ in water is 7.8 times smaller [1]), we have $H /\left(36 k_{B} T\right)$ $\simeq 0.45$, which is $\sim 126$ times smaller than the fitted $A_{1}$ $(\simeq 57)$. More accurate calculation of $U_{V}(r)$ for two spheres at separation $r_{0} \simeq 1.7 d$ gives [2] $U_{V}\left(r_{0}\right) / k_{B} T \simeq-0.034$, which is $\sim 10$ times smaller than the measured depth of the attractive well.

An alternative candidate is the electrostatic capillary attraction $[11,12,30-32]$. Because of the large difference in dielectric constant across the interface, there is an inhomogeneous pressure exerted on the interface, causing it to bend toward the water. The overlap of the deformed interface regions surrounding each sphere can result in an effective attraction of the form [12]

$$
U_{E}(r) \simeq-\frac{F^{2}}{2^{6} \pi \gamma}\left(\frac{d}{r}\right)^{6},
$$

where $\gamma(\simeq 72 \mathrm{dyn} / \mathrm{cm})$ is the surface tension of the waterair interface and $F=\widetilde{P}_{z}^{2} /\left(\pi \epsilon_{0} \epsilon d^{4}\right)$ is an effective force pulling the particle into the water. Using the fitted values of $q$ $=3300 e$ and $\lambda_{D}=96 \mathrm{~nm}$, we have $F^{2} /\left(2^{6} \pi \gamma k_{B} T\right) \simeq 4 \times 10^{-8}$, which is $10^{-9}$ times smaller than the fitted values of $A_{1}$. Oettel et al. [32] presented a more detailed analysis of the electrostatic capillary attraction. Their calculation suggested that the electrostatic capillary effect alone is probably too weak to explain the reported attraction between like-charged micrometer-sized particles if the colloidal systems under study were mechanically isolated (i.e., there is no external field involved).

Spontaneous fluctuations of counterions surrounding a test particle can generate correlated ion fluctuations for a nearby particle, giving rise to an attractive interaction analogous to the van der Waals attraction discussed above. This attraction has an approximate form [33],

$$
\frac{U_{F}(r)}{k_{B} T} \simeq-\frac{3 \Theta^{2}}{64}\left(\frac{d}{r}\right)^{6},
$$

where $\Theta^{2}<1$ is a function of $d / \lambda_{D}$. Similar to the van der Waals attraction, the attraction induced by thermal fluctuations of counterions is too small to account for the fitted values of $A_{1}$.

Other theoretical explanations [11-14] explore the effect of counterions beyond the usual Poisson-Boltzmann formulism when the surface separation of the particles becomes smaller than $\lambda_{D}$. The measurements shown in Fig. 4, however, clearly demonstrate that the observed attraction occurs at particle surface separations larger than $\lambda_{D}$ and thus cannot be explained by the theoretical models focused on the strongcoupling regime $[3,4,11-14]$.

\section{Attractions induced by surface-charge heterogeneity}

Another source of charge variations comes from heterogeneity of the charge distribution on the particle surface (quenched disorder). Such surface heterogeneity may develop either during the synthesis of the particles ("intrinsic heterogeneity") or during the ionization process in the aqueous phase ("kinetic heterogeneity"). For example, charged polystyrene spheres are found to have patchy domains of ionizable groups of size $\sim 100 \mathrm{~nm}$ on their surface $[18,19,21]$. These are intrinsic heterogeneities formed during the synthesis of the polystyrene spheres. While the silica spheres appear to have a macroscopically uniform surface, there are still many defects and impurity sites on the particle surface, as shown in Fig. 6(a) below. These naturally occurring surface defects and impurities can cause inhomogeneous ionization on the particle surface. The intrinsic and statistical fluctuations of the local surface charge density in turn introduce lateral inhomogeneities to the nearby counterion distribution. Regions with more surface charges attract more counterions and thus produce positionally correlated counterion localization in the vicinity of the particle surface $[34,35]$.

These initial surface charge inhomogeneities can be further amplified if salt is added into the water. It is known that the addition of salt enhances the ionization of the silica surface and hence the total surface charge of silica spheres increases with the salt concentration [26]. This is because the mixing of counterions $\left(\mathrm{H}^{+}\right.$and $\left.\mathrm{Na}^{+}\right)$in the vicinity of the 
silica surface causes a dilution of the local concentration of $\mathrm{H}^{+}$and thus allows the ionization reaction, $\mathrm{SiOH} \rightleftharpoons \mathrm{SiO}^{-}$ $+\mathrm{H}^{+}$, to proceed in a more positive direction. For an inhomogeneous surface, this increase in ionization is no longer uniform any more. Assuming that the local reaction rate $K$ $=\left[\Gamma_{\mathrm{SiO}^{-}}\right]\left[\mathrm{H}^{+}\right] /\left[\Gamma_{\mathrm{SiOH}}\right]$ remains unchanged and that the dilution effect of adding salt is the same across the entire surface, one can show [36] that regions with more surface charges will have a relatively larger increase in charge than those regions with less surface charges. Here $\left[\mathrm{\Gamma}_{\mathrm{SiO}^{-}}\right]$and $\left[\mathrm{H}^{+}\right] \mathrm{de}-$ note the surface density of the head groups $\Gamma_{\mathrm{SiO}^{-}}$and $\mathrm{H}^{+}$, respectively, and $\left[\Gamma_{\mathrm{SiOH}}\right]$ is the surface density of the chargeable $\mathrm{SiOH}$ sites. Therefore, the increase in surface ionization occurs more favorably in the patchy regions of surface charge, providing a positive feedback for the formation of larger patchy charge domains on the particle surface. To have a more quantitative and self-consistent description of the ionization process for an inhomogeneous silica surface, Huang and $\mathrm{Ng}$ [37] carried out a Monte Carlo simulation using a random local potential together with a formulism consistent with the Gouy-Chapman-Stern mean-field model [26]. The simulation results support the simplified argument given above and the interested readers are referred to Ref. [37] for more details.

The second effect of adding salt to the system is to increase the spatial resolution and contrast with which the surface charge inhomogeneity can be probed. This is because the smallest distance, over which the interparticle potential $U(r)$ is measured, is set by the screening length $\lambda_{D}$. Surface charge variations are measurable only when the size of the patchy domains becomes comparable or larger than $\lambda_{D}$. By continuously adding salt into the water, one reduces the value of $\lambda_{D}$ and thus increases the chance to detect the surface charge inhomogeneities.

\section{Atomic force microscopy measurements}

To examine the actual distribution of the surface charge groups and to see how it changes with the salt concentration, we conduct AFM measurements of surface topology of the individual silica spheres and phase shifts of the oscillating AFM cantilever. The AFM measurements are performed first on a dry layer of silica spheres adsorbed on a clean mica substrate. Figure 5(a) shows the height image (threedimensional) of a sphere's top surface in air (no water), which is smooth down to a few nanometers. The smooth top surface is seen more clearly in Fig. 5(c), which shows a cross-sectional view of the height image of the sphere's top surface. The three height profiles obtained in different azimuthal directions, as indicated by the three pairs of arrows shown in Fig. 5(b), overlap very well, indicating that the surface of the silica particles has a nice spherical shape. By further analyzing the cross-sectional profile of the height images, we find that the rms roughness of the particle surface is $\sim 0.36 \mathrm{~nm}$. Figure 5 thus suggests that the observed attractions shown in Fig. 4 are not likely to be caused by the shape- and surface-roughness-induced capillary attractions [38,39].

With the cantilever tip in the attractive regime $2-5 \mathrm{~nm}$ above a sphere's top surface, the phase delay $\varphi$ of the oscil-
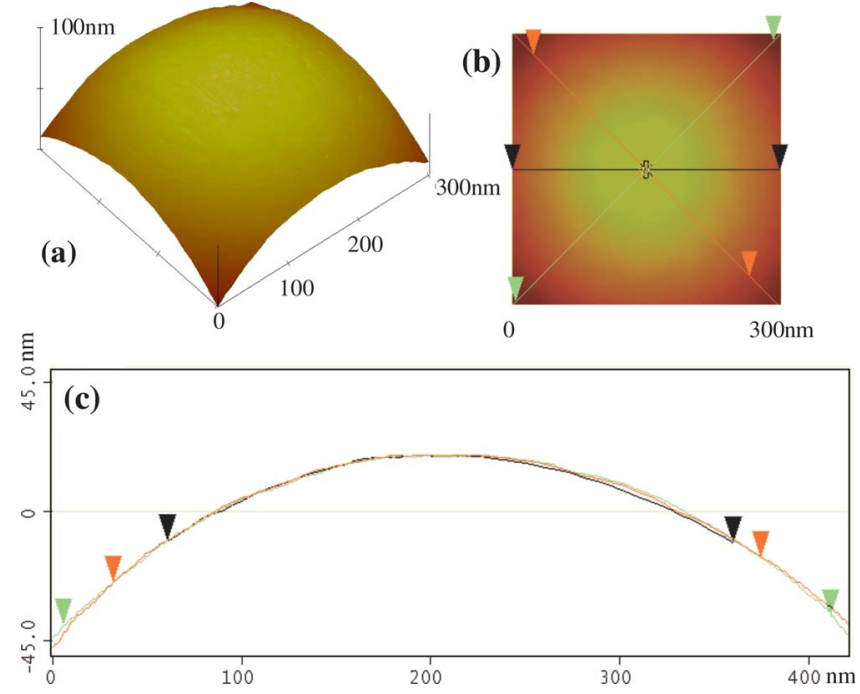

FIG. 5. (Color online) AFM measurements on dry silica spheres in air. (a) Three- and (b) two-dimensional AFM topographic images of a silica sphere in air (no water). (c) Cross-sectional view of the measured height image of the sphere's top surface. The height profiles are obtained in three different azimuthal directions as indicated by the three pairs of arrows of different colors (green, red, and black) shown in (b).

lating cantilever is determined primarily by the van der Waals attraction between the AFM tip and the almost flat top surface $[19,21,40]$. Figure 6(a) shows the phase image simultaneously recorded with Fig. 5(a). Phase fluctuations at the silica surface exhibit a granular pattern with grain size $\sim 10 \mathrm{~nm}$, and they distribute rather uniformly across the en-
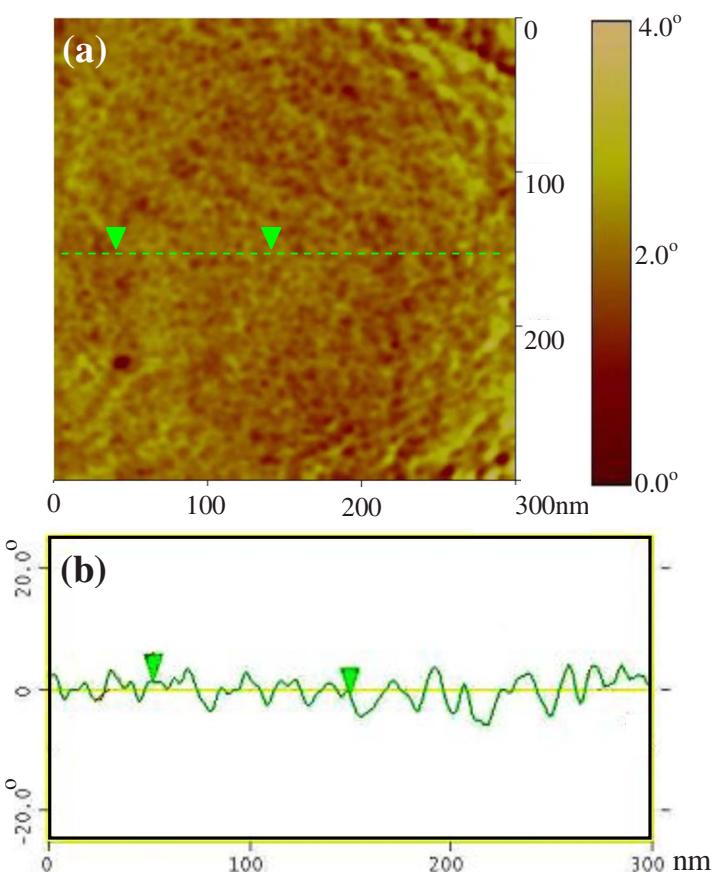

FIG. 6. (Color online) (a) AFM phase image of the sphere's top surface simultaneously recorded with Fig. 5(a). (b) Cross-sectional view of the measured phase image in an azimuthal direction as indicated by a pair of (green) arrows shown in (a). 


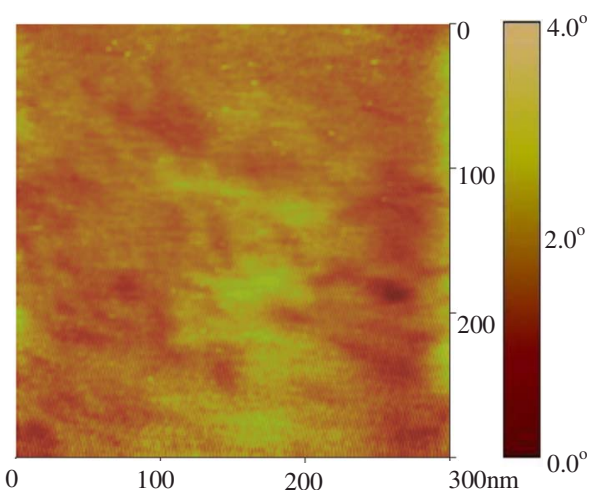

FIG. 7. (Color online) Measured AFM phase image of the top surface of a silica sphere fully immersed in a $1 \mathrm{mM} \mathrm{NaCl}$ solution.

tire view area. From the cross-sectional profile of the phase image shown in Fig. 6(b), we find that the rms value of the phase fluctuations is $\varphi_{\mathrm{rms}} \simeq 0.15^{\circ}$. This measurement indicates that the silica spheres have a rather uniform surface in air. By contrast, our early AFM measurements [18,19] showed that the polystyrene latex spheres have patchy domains on their surface with phase shifts as large as $\varphi \sim 50^{\circ}$.

Figure 7 shows the phase image of the top surface of a silica sphere fully immersed in a $1 \mathrm{mM} \mathrm{NaCl}$ solution. Patchy domains of size 50-100 nm appear with phase contrast up to $\varphi \sim 2^{\circ}$, which is 3-4 times larger than the phase fluctuations shown in Fig. 6(b). We believe that these patchy domains of irregular shape are associated with the nonuniform charge distribution on the particle surface [see Fig. 9(a) below]. While we were able to obtain a few AFM phase images, such as that shown in Fig. 7, the presence of water often causes problems for AFM imaging of the silica spheres. The van der Waals bonding between the individual silica sphere and mica substrate becomes very weak under water, making the sphere particularly vulnerable to small external perturbations, such as the scanning of an AFM tip. Slight motion of the sphere causes errors in the AFM imaging. This problem prevents us from conducting a systematic AFM study of the silica spheres in water.

To overcome the experimental difficulties, we conduct an alternative AFM study on a smooth and uniform silicon wafer under water with varying salt concentrations. The silicon wafer used has a thin oxidized layer $\left(\mathrm{SiO}_{2}\right)$ of thickness $\sim 20 \mathrm{~nm}$ on the surface and its rms surface roughness is 0.05 $\mathrm{nm}$. This surface is chosen because its ionization kinetics under water is the same as that of the silica spheres and it does not have other unwanted surface complications, such as pre-existing patchiness. Being a large flat surface, the oxidized silicon wafer provides experimental convenience for systematic AFM measurements under water. The controlled AFM measurements on the silicon surface are conducted in the same salt concentration range $(0-100 \mu \mathrm{M})$, and here we show some sample AFM images. Figures 8(a) and 8(b) compare the phase images obtained in fresh deionized water and in a $100 \mu \mathrm{M} \mathrm{NaCl}$ solution. Similar to Fig. 7, patchy domains of size $\sim 100 \mathrm{~nm}$ form in the salt solution with phase contrast $\varphi \sim 0.7^{\circ}$, which is approximately nine times larger than the random-phase fluctuations shown in Fig. 8(a).

To further characterize the interaction between the AFM tip, which is made of silicon nitride $\left(\mathrm{Si}_{3} \mathrm{~N}_{4}\right)$, and the oxidized
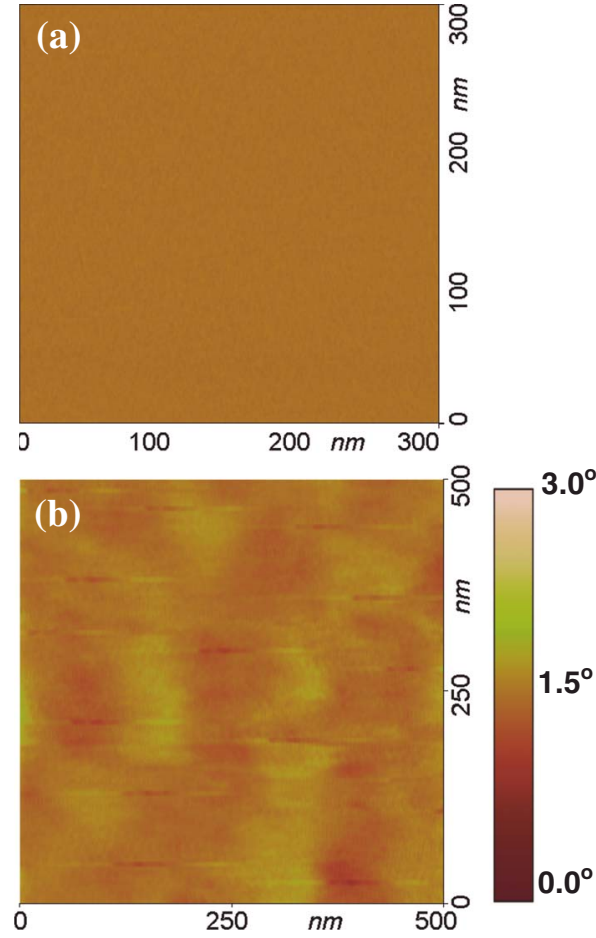

FIG. 8. (Color online) Measured AFM phase images of an oxidized silicon wafer in (a) fresh deionized water and (b) $100 \mu \mathrm{M}$ $\mathrm{NaCl}$ solution.

silicon surface, we conduct force-volume imaging and obtain a space-resolved two-dimensional array of force-distance curves over a specified area. Figure 9(a) shows the AFM force amplitude image when the AFM tip is kept at a fixed distance approximately $5 \mathrm{~nm}$ above the oxidized silicon surface. At this distance, the force between the AFM tip and the silicon surface is usually attractive due to van der Waals attraction [20,40], and yet we find repulsive domains of size $\sim 100 \mathrm{~nm}$ in the salt solution. These repulsive domains are displayed as brighter regions in Fig. 9(a) and the color code only shows the force contrast (with the maximum attractive force being set as zero). In these brighter regions the Coulomb repulsion between the weakly charged AFM tip and the silicon surface (both are negatively charged) overcomes the van der Waals attraction.

Figure 9(b) shows two representative force curves as a function of the distance $z$ between the silicon nitride tip and oxidized silicon surface. The solid curve is obtained at a location in the dark region and the dashed curve is obtained in a bright region. In the separation range $5 \leqq z \leqq 7 \mathrm{~nm}$, one finds the usual van der Waals attraction in the dark region, whereas in the bright region the interaction force is always repulsive. Figures 9(a) and 9(b) thus clearly reveal that the attractive and repulsive interactions coexist on the oxidized silicon surface. The above AFM measurements suggest that the added salt can indeed produce patchy charges on the smooth silicon surface. This mechanism is also applicable to the silica surface because its ionization kinetics under water is the same as that of the oxidized silicon surface. 

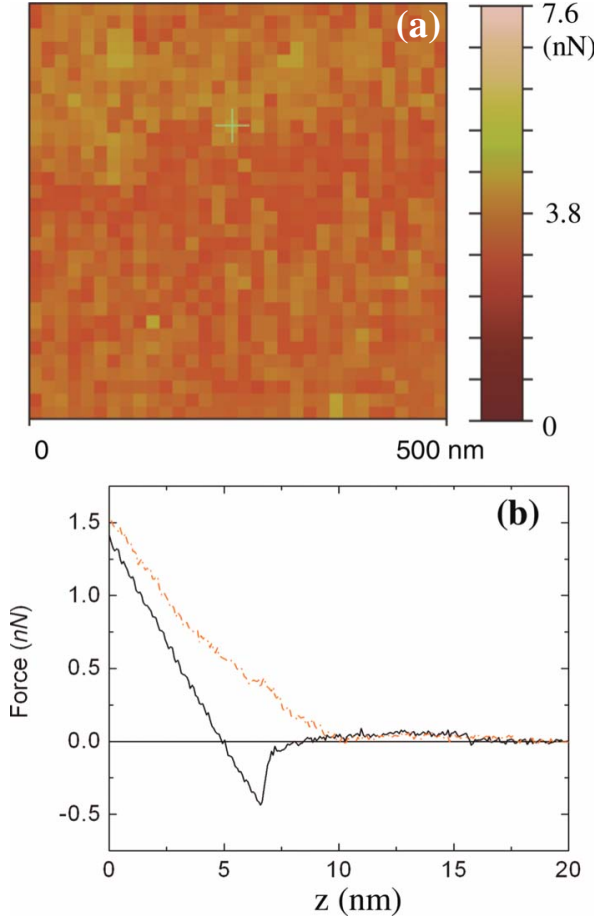

FIG. 9. (Color online) (a) Measured AFM force amplitude image of the oxidized silicon wafer in a $100 \mu \mathrm{M} \mathrm{NaCl}$ solution. (b) Two representative force curves as a function of the distance $z$ between the silicon nitride tip and oxidized silicon surface. The solid curve is obtained in a dark region and the dashed curve is obtained in a bright region shown in (a).

\section{E. Electrostatic potential of a charged interfacial particle}

To calculate the electrostatic potential produced by an interfacial particle with patchy surface charges, one may envision to divide the particle surface in contact with the water into small pieces, each has a surface charge $-q^{\prime}$. The counterions in water have a net charge $+q^{\prime}$ at an average distance $\lambda_{D}$ (the screening length) from the sphere's surface. Therefore, each piece of the particle surface can be treated as a small dipole with its direction normal to the local surface and amplitude proportional to $q^{\prime} \lambda_{D}$ [29]. This situation is depicted in Fig. 10, in which we use short (red) solid arrows to indicate the surface dipoles. For a uniformly charged particle (i.e., the solid arrows shown in Fig. 10 are uniformly distributed over the particle surface in contact with water), its effective dipole moment after adding up all small dipoles on the surface is always perpendicular to the interface. The horizontal components are canceled out with each other because of the spherical symmetry. For a nonuniformly charged particle (i.e., the spatial distribution of the solid arrows shown in Fig. 10 is not uniform), however, the horizontal components cannot be canceled out any more, giving rise to an effective dipole moment with both a permanent vertical component (shown as a vertical dashed arrow in Fig. 10) and a horizontal component (shown as a horizontal dashed arrow in Fig. $10)$, which rotates randomly in the plane of the interface due to the Brownian rotation of the particle $[19,28]$.

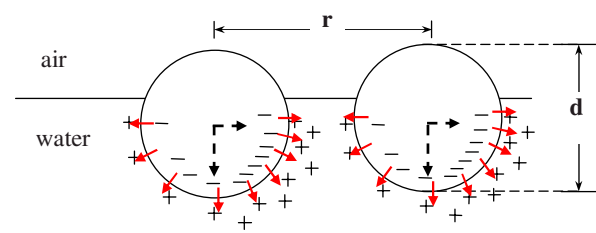

FIG. 10. (Color online) Schematic of charged particles at the water-air interface and distributions of the surface charges and counterions near the interface. The short (red) solid arrows indicate the surface dipoles. The dashed arrows indicate the vertical and horizontal components of the resulting effective dipole moment of the particle.

As mentioned earlier, the interaction potential between two permanent out-of-plane (vertical) dipoles gives rise to the $1 / r^{3}$ repulsion shown in Eq. (4). The interaction potential between two in-plane (horizontal) dipoles has the form $V\left(r, \phi_{1}, \phi_{2}\right) \sim f\left(\phi_{1}, \phi_{2}\right) / r^{3}$, where $r$ is the interparticle separation and $f\left(\phi_{1}, \phi_{2}\right)=3 \cos \left(\phi_{1}\right) \cos \left(\phi_{2}\right)-\cos \left(\phi_{2}-\phi_{1}\right)$ describes the alignment between the two in-plane dipoles whose orientations are specified by the angles $\phi_{1}$ and $\phi_{2}$ with respect to the vector connecting the two particles $[19,28]$. The effective interaction potential $U_{D}(r)$ between two randomly orientated in-plane dipoles is given by

$$
e^{-U_{D}(r) / k_{B} T}=\left\langle e^{-V\left(r, \phi_{1}, \phi_{2}\right) / k_{B} T}\right\rangle,
$$

where $\langle\ldots\rangle$ represents a thermal average over the two angles $\phi_{1}$ and $\phi_{2}$. By expanding $\left\langle\exp \left[-V\left(r, \phi_{1}, \phi_{2}\right) / k_{B} T\right]\right\rangle$ in power series and using the fact that $\left\langle f\left(\phi_{1}, \phi_{2}\right)\right\rangle=0$, we have $U_{D}(r) / k_{B} T \simeq\left\langle\left[V\left(r, \phi_{1}, \phi_{2}\right) / k_{B} T\right]^{2}\right\rangle \sim\left\langle f^{2}\left(\phi_{1}, \phi_{2}\right)\right\rangle / r^{6}$, which gives a $1 / r^{6}$ attraction [1].

The final result for $U_{D}(r) / k_{B} T$ is

$$
\frac{U_{D}(r)}{k_{B} T} \simeq-\frac{4\left\langle P_{\|}^{2}\right\rangle^{2}}{\left(4 \pi \epsilon \epsilon_{0} k_{B} T d^{3}\right)^{2}}\left(\frac{d}{r}\right)^{6},
$$

where $\left\langle P_{\|}^{2}\right\rangle$ is the mean-squared value of the fluctuating dipole moment. For $N$ patchy domains randomly distributed on the particle surface, we have $\left\langle P_{\|}^{2}\right\rangle \simeq\left(q \lambda_{D}\right)^{2} / N$ and thus $A_{1} \simeq\left[2\left(q \lambda_{D}\right)^{2} /\left(4 \pi \epsilon \epsilon_{0} k_{B} T d^{3} N\right)\right]^{2}$. Using $\quad\left(\lambda_{D}, q\right)$ $=(96 \mathrm{~nm}, 3300 e)$ and $A_{1}=57$, we find $N \simeq 49$. The mean separation between two patchy domains is given by $\ell$ $\gtrsim\left(\pi d^{2} / 2 N\right)^{1 / 2} \simeq 130 \mathrm{~nm}$, which is larger than $\lambda_{D}$ as expected. From Fig. 8 we find that the obtained value of $N$ gives a correct estimate for typical domain separations.

In obtaining Eq. (9), we have made several assumptions. First, for simplicity, we only considered the interaction between two fluctuating in-plane dipoles. For particles containing many small charge patches, one can first write down an effective dipole-dipole interaction between two nearby patches and then carry out an average over all particle orientations (i.e., over all patches) to get an effective interaction 
potential between two particles. Such an average will give the same $1 / r^{6}$ dependence as discussed above. Second, in the power expansion of Eq. (8), we have assumed that $U_{D}(r) / k_{B} T<1$. This approximation is justified as the maximum attraction shown in Fig. 4 is only approximately $0.3 k_{B} T$.

The third approximation we made is that the dipole-dipole interactions at the interface are not screened. Hurd has showed that the interaction between the induced out-of-plane dipoles at the interface is not screened [29]. In a recent calculation, $\mathrm{Ng}$ and Zhou [28] showed that this is also true for in-plane dipoles at the interface. This is because a part of the electric field generated by the surface charges and the surrounding counterions can go through the air phase, which has no ion to screen out the electric field [27,28]. In a recent experiment, Polin et al. [17] showed that coating the waterglass interface with a conducting gold layer eliminates the confinement-induced attractions at the interface.

Finally, in the above discussion we have not considered the possible pinning effect of the interface in the immediate vicinity of the contact line between the interface and particle. Pinning of the interface to surface heterogeneity can have two effects on the interfacial particles. First, it may restrict the Brownian rotation of the particles at the interface because of the extra drag force generated by the moving contact line [41]. In studies of the particle mobility at an interface, one usually assumes that the particles can freely rotate about the vertical axis perpendicular to the interface and their rotation about the other axes will be severely damped out [41]. Such a random rotation in the plane of the interface is adequate to provide the thermal averaging in Eq. (8). The second effect of the interface pinning is to introduce meniscus deformation near the contact line, which would give rise to another source of capillary interaction. At the moment, we do not have an independent means to quantify how large this effect would be [42] and a further study is needed. However, because the estimated number of the patchy charge domains per particle is large $(N \simeq 49)$, one would expect that the resulting capillary interaction after the thermal averaging will be isotropic. This conclusion does not agree with the experimental observation that the interparticle potential at the interface is more likely to be anisotropic (see the discussion in Sec. III F below for more details).

As discussed in Sec. III A, the minimum position $r_{0}$ of the attractive well, which determines the interparticle distance of the particle clusters observed in early experiments [9-11], is determined primarily by the balance between the screened Coulomb repulsion and the attraction proposed here. As a result, the equilibrium interparticle separation will depend on the actual interaction parameters involved in each colloidal system. The early experiments were conducted in colloidal systems different from the present one in several ways. First, most of the early experiments were conducted in salt-free aqueous solutions or in an oil-dominant phase, in which the value of the screening length $\lambda_{D}$ is much larger than that in the present experiment. Second, the interaction amplitude also varies a lot among different colloidal systems. For those experiments using strongly charged polystyrene latex spheres, both the depth of the attractive well at $r_{0}$ and the height of the repulsive barrier at $r_{1}\left(>r_{0}\right)$ can be much larger than a few $k_{B} T$ 's so that the particles can choose to either stay in the deep attractive well forming stable clusters $[18,19]$ or remain outside the repulsive barrier at a larger interparticle separation forming ordered or disordered arrays of particles under the confinement of a fixed area [19,27].

For weakly charged silica spheres, because the depth of the attractive well and the height of the repulsive barrier are both smaller than a $k_{B} T$ as shown in Fig. 4(b), no stable particle cluster or repulsive particle array is formed. Such a weakly interacting system, however, allows us to map out the whole pair-correlation curve $g(r)$ [or equivalently the interaction potential curve $U(r)]$ from the measured equilibrium particle configurations. In an early experiment, Han and Grier [8] measured $g(r)$ for a monolayer of silica spheres in water near a glass plate. They found an attractive interparticle potential with an interaction range similar to that shown in Fig. 4(b). Other early experiments $[5,9,10]$, however, only reported the formation of colloidal clusters, and no systematic measurement of the interaction parameters was provided, making the quantitative comparison with theoretical models difficult. In fact, an important purpose of the present experiment is to provide detailed measurements of the interaction parameters and their dependence on the experimental control parameters so that one can have a quantitative comparison with theory, such as that shown in Eq. (4).

\section{F. Anisotropy of the interaction potential between the interfacial particles}

While the dipole potential $U_{D}(r) / k_{B} T$ shown in Eq. (9) proves instructive for our purpose, this model nevertheless is phenomenological and is valid only at the large $r / d$ (point dipole) limit. Because the attractive interactions observed here occur at only one or two particle diameters away but not at the large $r / d$ limit and they are observed at a finite screening length but not at the small screening length limit (i.e., salty water is not a metal), care has to be taken when comparing theories of asymptotic expansion with the actual experiment. From Fig. 4(b) one finds that the measured $U(r)$ is more complicated; it contains at least three parts: (i) an exponential-like steep repulsion at small $r(r \lesssim 1.7 d)$, (ii) an attraction dominated range $1.7 d \lesssim r \lessgtr 2.3 d$, and (iii) a slow decaying repulsion at large $r(r \gtrsim 2.3 d)$. (This estimation is made based on the measured $U(r)$ at $C \simeq 50 \mu \mathrm{M}$.) Given the fact that there are three different types of interactions in a narrow $r$ range from $\sim 1.5 d$ to $(4-5) d$, separating the measured $U(r)$ into three different asymptotic forms is neither practical nor reliable.

In the present experiment, we choose a different way to test the theory; namely, we measure the changes of $U(r)$ by systematically varying the salt concentration $C$. This allows us to see which force operates in what range of the interparticle separations and at the same time to avoid possible influence of experimental artifacts, which do not change with $C$. By careful examination of Eq. (4), one finds that the three terms in Eq. (4) operate in different length scales. The first term (screened Coulomb repulsion) changes sensitively with the salt concentration. This term fits well the small- $r$ portion of the measured $U(r)(r \lesssim 1.7 d)$ with the expected interaction 
range $\lambda_{D}$ (Debye screening length). With this fitting we immediately conclude that the long-range attraction and repulsion observed for $r \gtrsim 1.7 d$ are not screened. If they were screened, an exponential factor, $\exp \left(-r / \lambda_{D}\right)$, has to be multiplied to the second and third terms in Eq. (4), resulting in two terms which decay even faster than the first term and thus do not fit the data at all.

The third term in Eq. (4) is a classical term for the dipoledipole repulsion, which has been verified in recent experiments at the water-air interface $[19,27]$. This term fits well the large- $r$ portion of the measured $U(r)$ for $r \gtrsim 2.3 d$. This interaction range does not change with the salt concentration. Both the first and third terms in Eq. (4) are the standard potentials for the interfacial particles, which have been tested in previous experiments $[1,2,19,24,27]$.

The new finding of the experiment is the attractive part of the measured $U(r)$, which is located primarily in the intermediate range, $1.7 d \lesssim r \lessgtr 2.3 d$, between the short-range screened Coulomb repulsion and the long-range dipoledipole repulsion. Because the particles are only one or two diameters away from each other, higher-order corrections at finite interparticle separations may exist, making it difficult to distinguish different physical mechanisms by simple power counting of the fitted power-law curve. Clearly, other types of measurement are needed in order to further pinpoint the physical origin of the observed attraction.

In Sec. III D, we described the AFM measurements on the silica and oxidized silicon surfaces. These measurements suggest that the main effect of adding salt is to introduce patchy charge domains on the particle surface. This finding together with the optical observation of chainlike structure formation (to be described below) prompt us to consider anisotropic attractions of electrostatic origin rather than the usual isotropic interactions, such as capillary forces and attractions resulting from wetting and surface roughness of the particles, for the interfacial particles. Dipole-dipole attractions tend to cause three or more particles to form chainlike structures. For polystyrene latex spheres, which exhibit a higher degree of surface heterogeneity, the anisotropic nature of the interparticle attraction of amplitude larger than a few $k_{B} T$ has been confirmed by the direct observation of chainlike structures at the interface [18] and by the recent measurement of three-body interactions between a dimer and a particle using optical tweezers [43].

For silica spheres, because the interactions are weak $\left(<k_{B} T\right)$, the particles at the salty water interface form transient chainlike structures, as shown in Fig. 11. These chainlike structures come and go, which is a hallmark of weak dipolar fluids. By contrast, the particles at the pure water interface form random-looking structures, as shown in Fig. 1. Perhaps, one may see these fluctuating chainlike structures more clearly from the online supplement movie [44] by repeated pause and play of the movie to view the intermediate particle configurations. These observations further support our conclusion that salt ions promote the formation of patchy charges at the particle surface, leading to a dipolelike attraction between the interfacial particles. The proposed dipole interaction is the lowest-order electrostatic attraction, which goes as $1 / r^{3}$ and becomes $1 / r^{6}$ after thermal averaging over the orientations of two fluctuating in-plane dipoles. Cer-

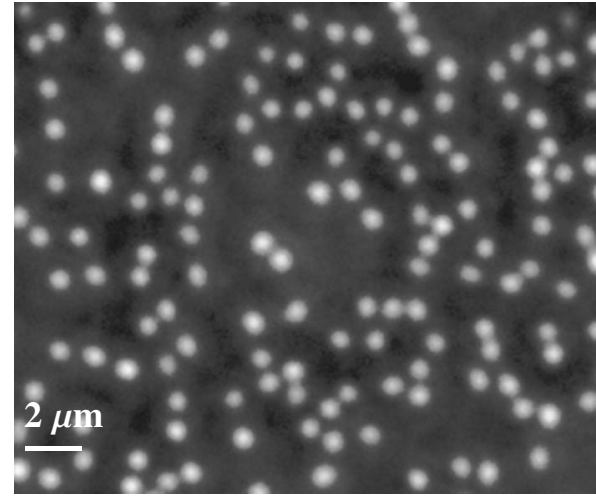

FIG. 11. Typical configuration of the silica spheres at the waterair interface with $\sim 15 \mu \mathrm{M}$ salt ions in the aqueous phase. The measurement is made at the area fraction $n \simeq 0.11$.

tainly, a more quantitative analysis is needed to further characterize the anisotropy of the measured attraction.

\section{G. Imaging errors}

While Fig. 4 clearly shows a systematic change in $U(r)$ with increasing salt concentration $C$, the interaction amplitude observed is nevertheless small compared with the thermal energy $k_{B} T$. Therefore, one needs to pay special attention to the experimental uncertainties of the measured $g(r)$. Recently, Baumgartl et al. [15] and Ramirez-Saito et al. [16] showed that small distortions in the optical images of neighboring particles may produce small errors in the measured interparticle distance, leading to artificial features in the resulting $g(r)$. In this section, we show that systematic errors on the particle separation due to the overlap of their optical images, which have been studied for bright field microscopy $[15,16]$, are very small in our case and do not affect the results shown in Fig. 4.

As mentioned earlier, phase contrast microscopy is used in this experiment to obtain good images of the silica spheres. Figure 12(a) shows the phase contrast image of a silica sphere at the water-air interface. The magnification of the objective used is $60 \times$. Figure 12 (b) shows the azimuthally averaged intensity profile $I(x)$ of the silica particle as a function of the radial distance $x$ away from the sphere's center. The main difference between this intensity profile and that obtained by bright field microscopy is that there is a small shoulder peak on each side of the main peak, which gives rise to an intensity surplus, whereas in the bright field microscopy the shoulder peak becomes valley, resulting in an intensity deficit.

Figure 13(a) shows the overlap of two identical intensity profiles, $I(x)-I_{0}$, (red and green) and the resultant profile (black) of the two particles. Each individual intensity profile, $I(x)-I_{0}$, is taken from Fig. 12(b) with the background $I_{0}$ subtracted out. When two such phase contrast images overlap, the shoulder peak of the left particle (red curve) adds an intensity bias to the intensity profile of the right particle (green curve), causing the intensity weighted centroid of the right particle to shift to the left. As a result, the measured 

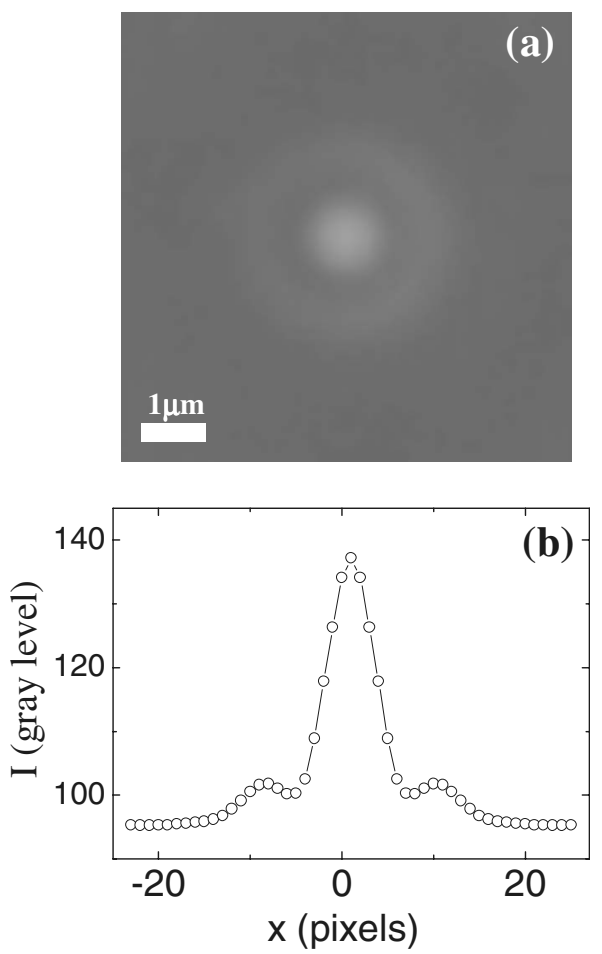

FIG. 12. (a) Phase contrast image of a silica sphere at the waterair interface. The magnification of the objective used is $60 \times$. (b) Azimuthally averaged intensity profile $I(x)$ of the silica particle as a function of the radial distance $x$ away from the sphere's center.

interparticle distance $r_{m}$ is smaller than the actual separation $r$ of the two particles.

This effect explains Fig. 13(b), which shows the deviation of the interparticle distance, $\Delta r=r_{m}-r$, as a function of the measured separation $r_{m}$. In the plot, both $\Delta r$ and $r_{m}$ are normalized by the particle diameter $d$. The value of $\Delta r$ is obtained by (i) obtaining an average intensity profile of the imaged particles [as shown in Fig. 12(b)], (ii) placing two such intensity profiles at a given separation $r$ (which is known in this case), (iii) calculating the intensity weighted $r_{m}$ using the same algorithm as that used in the experiment, and (iv) finding the deviation $\Delta r=r_{m}-r$ for different values of $r$. This procedure has been used in previous studies [15-17] to estimate the imaging errors.

The effect of $\Delta r$ on the pair-correlation function $g(r)$ can be estimated using the equation [45]

$$
g(r) d r=g\left(r_{m}\right) d r_{m},
$$

where $g\left(r_{m}\right)$ is the measured pair-correlation function with the interparticle distance $r_{m}$ and $g(r)$ is the artifact-free paircorrelation function. While for simplicity this equation was derived for a monotonic function in some textbooks, the validity of this equation is, in fact, quite general. Because this is a differential equation, only local monotone is used in the derivation and no global monotone is required [46]. For dilute particle suspensions, distortions due to the three-body overlap can be ignored, and Eq. (10) yields
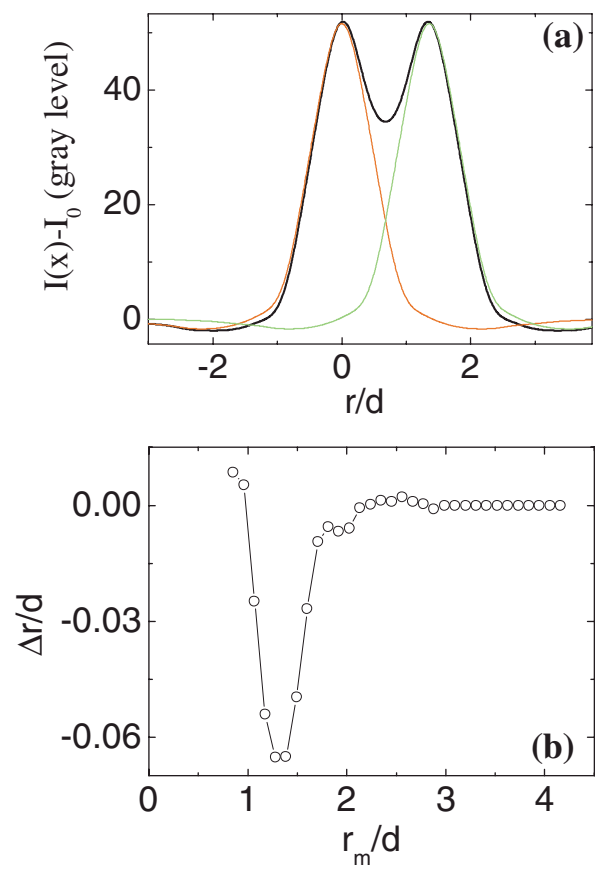

FIG. 13. (Color online) (a) Overlap of two identical intensity profiles, $I(x)-I_{0}$, (red and green) and the resultant profile (black) of the two particles. Each individual intensity profile, $I(x)-I_{0}$, is taken from Fig. 12(b) with the background $I_{0}$ subtracted out. (b) Obtained deviation of the interparticle distance, $\Delta r=r_{m}-r$, as a function of the measured separation $r_{m}$. In the plot, both $\Delta r$ and $r_{m}$ are normalized by the particle diameter $d$.

$$
g(r)=g\left(r_{m}\right) \frac{d r_{m}}{d r}
$$

When the area fraction $n$ of the particle sample is small (which is true in our case), one has

$$
\frac{U(r)}{k_{B} T} \simeq-\ln [g(r)]=\frac{U\left(r_{m}\right)}{k_{B} T}-\ln \left(\frac{d r_{m}}{d r}\right)
$$

Therefore, one finds the deviation of the interaction potential, $\Delta U / k_{B} T=\left[U(r)-U\left(r_{m}\right)\right] / k_{B} T=-\ln \left(d r_{m} / d r\right)$.

Figure 14 shows the measured $d r_{m} / d r$ as a function of $r_{m} / d$ for silica spheres at the water-air interface. Because the

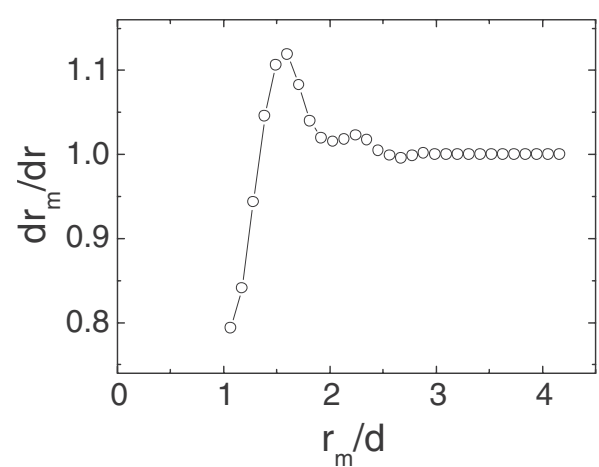

FIG. 14. Measured $d r_{m} / d r$ as a function of $r_{m} / d$ for silica spheres at the water-air interface. 


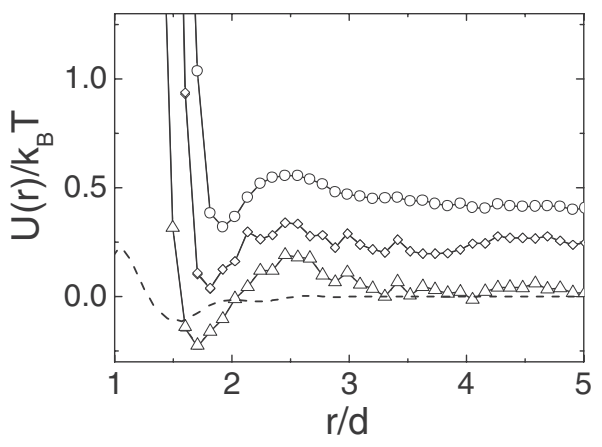

FIG. 15. Comparison between the calculated potential deviation $\Delta U / k_{B} T$ (dashed line), the directly measured $U\left(r_{m}\right) / k_{B} T$ (solid lines), and the corrected potential $U(r) / k_{B} T$ (symbols). The solid lines show the same data in Fig. 4(b).

value of $d r_{m} / d r$ depends on the experimental details, such as magnification, focal plan and type of optical microscopy used, one needs to evaluate $d r_{m} / d r$ case by case. With the measured $d r_{m} / d r$ shown in Fig. 14, we calculate the potential deviation $\Delta U / k_{B} T \simeq-\ln \left(d r_{m} / d r\right)$ (dashed line) and compare it with the directly measured $U\left(r_{m}\right) / k_{B} T$ (solid line) as shown in Fig. 15. It is seen that small deviations in the pair potential occur only in the range $r \lessgtr 1.5 d$, in which the measured $U\left(r_{m}\right) / k_{B} T$ is dominated by a strong Coulomb repulsion. In Fig. 15, we also compare the "corrected" potential $U(r) / k_{B} T=U\left(r_{m}\right) / k_{B} T+\Delta U / k_{B} T$ (symbols) with the directly measured $U\left(r_{m}\right) / k_{B} T$ (solid lines). The two sets of data overlap so well that they are almost indistinguishable with each other. Figure 15 thus demonstrates that the artifacts caused by the image overlap between two neighboring particles are negligibly small in our experiment and that they do not affect the results shown in Fig. 4.

It should be pointed out that the systematic errors due to the particle image overlap are caused by the interference of light and have nothing to do with the experimental control parameters, such as the salt concentration $C$. In the experiment, we measured $U(r)$ for 12 different values of $C$ in the range 0-200 $\mu \mathrm{M}$. Systematic changes in $U(r)$ are observed with increasing $C$ while keeping all other experimental conditions unchanged. The well-controlled measurements shown in Figs. 3 and 4, therefore, effectively eliminate the possibility of experimental artifacts.

\section{CONCLUSION}

We have carried out a systematic study of interactions between weakly charged silica spheres at the water-air interface. The silica spheres used in the experiment have a diameter $d=0.73 \pm 0.04 \mu \mathrm{m}$ and their surface charge density $\sigma$ $\simeq 0.01 \mu \mathrm{C} / \mathrm{cm}^{2}$, which is approximately 200 times smaller than that of polystyrene latex spheres. Both particles are representative of the charged particles commonly used in colloidal science. In the experiment, we used optical microscopy to measure the pair-correlation function $g(r)$ with varying concentrations of salt $(\mathrm{NaCl})$ added in the aqueous phase. From the measured $g(r)$, we obtain the interaction potential $U(r)$ between the interfacial particles. The addition of salt reduces the repulsive range of the measured $U(r)$ considerably. Such a reduction in the screening range opens up a window of length scales, in which one could observe weaker interactions. As shown in Fig. 4(b), the measured $U(r)$ contains three parts: (i) an exponential-like steep repulsion at small interparticle separation $r(r \lessgtr 1.7 d)$, (ii) an attraction dominated range $1.7 d \leqslant r \leqslant 2.3 d$, and (iii) a slow decaying repulsion at large values of $r(r \geqslant 2.3 d)$. The balance between the first two terms gives rise to an attractive well of approximately $-0.3 k_{B} T$ in amplitude at $r_{0} \simeq 1.7 d$. The sum of the last two terms produces a repulsive barrier of $\sim 0.15 k_{B} T$ in amplitude at $r_{1} \simeq 2.5 d$.

It is found that the measured $U(r)$ can be well described by a sum of three interaction potentials as shown in Eq. (4). The first term is the screened Coulomb repulsion, which is the leading-order potential in the aqueous phase $[1,2]$. The second term is the $1 / r^{3}$ repulsion resulting from the interaction between the induced out-of-plane dipoles at the interface $[24,29]$. The third term is a $1 / r^{6}$ attraction, which is located in the intermediate range between the short-range Coulomb repulsion and the long-range dipole-dipole repulsion. There are several candidates for the origin of the attraction with a $1 / r^{6}$ dependence at the large $r / d$ limit. They include the van der Waals attraction [1], the electrostatic capillary attraction $[11,12,30-32]$, and a van-der-Waals-type attraction due to correlated fluctuations of counterions between two nearby particles [33]. Using the known parameters involved, we calculate the amplitude of each attraction and find that these attractive forces are simply too small (at least $10^{-2}$ times smaller) to account for the amplitude of the measured attraction.

Another source of charge variations comes from heterogeneity of the charge distribution on the particle surface (quenched disorder). Such surface heterogeneity may develop either during the synthesis of the particles (intrinsic heterogeneity) or during the ionization process in the aqueous phase (kinetic heterogeneity). To examine the spatial distribution of the surface charge groups and to see how it changes with the salt concentration, we conducted atomic force microscopy (AFM) measurements on the surface of the individual silica spheres and on the smooth oxidized silicon wafer. The two surfaces share the common ionization kinetics. AFM phase and force-volume images show that patchy domains of size $50-100 \mathrm{~nm}$ form on the silica and oxidized silicon surfaces when they are in contact with the salty solution. These AFM measurements indicate that the surface ionization in water is not uniform as is commonly believed. Such patchy charges can introduce fluctuating in-plane dipoles, leading to an attraction at short interparticle distances and formation of (fluctuating) chainlike structures at the interface.

Charge heterogeneity on the colloidal surface is responsible for the attractive interactions observed in both the strongly charged polystyrene spheres and weakly charged silica spheres at a water-air interface and possibly, in a more general way, for attractive interactions seen in other systems that involve confinement by a wall or interface. The surface heterogeneity is manifested most effectively at the interface, 
at which the particle interaction becomes very sensitive to the asymmetry of the surface charge distribution. While the proposed mechanism proves instructive for our purpose, it is nevertheless a phenomenological model. A more quantitative analysis is certainly needed and the present work represents the first step toward this direction.

\section{ACKNOWLEDGMENTS}

We are grateful for useful discussions with P. Chaikin, D. Weitz, and H.-K. Tsao. This work was supported by Research Grants Council of Hong Kong SAR (Grants No. HKUST602806 and No. HKUST602803) and the U.S. National Science Foundation (Grant No. EPS-0132534).
[1] J. Israelachvili, Intermolecular and Surface Forces, 2nd ed. (Academic Press, San Diego, 1991).

[2] W. B. Russel, D. A. Saville, and W. R. Schowalter, Colloidal Dispersion (Cambridge University Press, Cambridge, UK, 1989).

[3] P. Attard, Curr. Opin. Colloid Interface Sci. 6, 366 (2001).

[4] D. G. Grier and Y. Han, J. Phys.: Condens. Matter 16, S4145 (2004).

[5] N. Ise, T. Okubo, M. Sugimura, K. Ito, and H. J. Nolte, J. Chem. Phys. 78, 536 (1983).

[6] G. M. Kepler and S. Fraden, Phys. Rev. Lett. 73, 356 (1994).

[7] M. D. Carbajal-Tinoco, F. Castro-Román, and J. L. ArauzLara, Phys. Rev. E 53, 3745 (1996).

[8] Y. Han and D. G. Grier, Phys. Rev. Lett. 91, 038302 (2003); J. C. Crocker and D. G. Grier, Phys. Rev. Lett. 77, 1897 (1996).

[9] F. Ghezzi and J. C. Earnshaw, J. Phys.: Condens. Matter 9, L517 (1997).

[10] J. Ruiz-Garcia, R. Gámez-Corrales, and B. I. Ivlev, Phys. Rev. E 58, 660 (1998).

[11] M. G. Nikolaides, A. R. Bausch, M. F. Hsu, A. D. Dinsmore, M. P. Brenner, C. Gay, and D. A. Weitz, Nature (London) 420, 299 (2002).

[12] M. Megens and J. Aizenberg, Nature (London) 424, 1014 (2003).

[13] T. A. Angelini, H. Liang, W. Wriggers, and G. C. L. Wong, Proc. Natl. Acad. Sci. U.S.A. 100, 8634 (2003).

[14] W. M. Gelbart, R. F. Bruinsma, P. A. Pincus, and V. A. Parsegian, Phys. Today 53 (9), 38 (2000).

[15] J. Baumgartl and C. Bechinger, Europhys. Lett. 71, 487 (2005).

[16] A. Ramirez-Saito, C. Bechinger, and J. L. Arauz-Lara, Phys. Rev. E 74, 030401(R) (2006).

[17] M. Polin, D. G. Grier, and Y. Han, Phys. Rev. E 76, 041406 (2007).

[18] W. Chen, S. Tan, T. K. Ng, W. T. Ford, and P. Tong, Phys. Rev. Lett. 95, 218301 (2005).

[19] W. Chen, S. Tan, Z.-H. Huang, T. K. Ng, W. T. Ford, and P. Tong, Phys. Rev. E 74, 021406 (2006).

[20] J. D. Feick and D. Velegol, Langmuir 18, 3454 (2002).

[21] S. Tan, R. L. Sherman, Jr., D. Qin, and W. T. Ford, Langmuir 21, 43 (2005).

[22] Langmuir-Blodgett Troughs Operating Manual, 6th ed. (Nima Technology Ltd., UK, 2001).

[23] S. H. Behrens and D. G. Grier, Phys. Rev. E 64, 050401(R)
(2001).

[24] P. Pieranski, Phys. Rev. Lett. 45, 569 (1980).

[25] G. Tolnai, A. Agod, M. Kabai-Faix, A. L. Kovács, J. J. Ramsden, and Z. Hórvölgyi, J. Phys. Chem. B 107, 11109 (2003).

[26] S. H. Behrens and D. G. Grier, J. Chem. Phys. 115, 6716 (2001).

[27] R. Aveyard, B. P. Binks, J. H. Clint, P. D. I. Fletcher, T. S. Horozov, B. Neumann, V. N. Paunov, J. Annesley, S. W. Botchway, D. Nees, A. W. Parker, A. D. Ward, and A. N. Burgess, Phys. Rev. Lett. 88, 246102 (2002).

[28] T. K. Ng and Y. Zhou, e-print arXiv:cond-mat/0703667.

[29] A. J. Hurd, J. Phys. A 18, L1055 (1985).

[30] L. Foret and A. Würger, Phys. Rev. Lett. 92, 058302 (2004).

[31] K. D. Danov, P. A. Kralchevsky, and M. P. Boneva, Langmuir 20, 6139 (2004).

[32] M. Oettel, A. Dominguez, and S. Dietrich, Phys. Rev. E 71, 051401 (2005).

[33] Y.-J. Sheng and H. K. Tsao, Phys. Rev. E 69, 060401(R) (2004).

[34] D. B. Lukatsky, S. A. Safran, A. W. C. Lau, and P. Pincus, Europhys. Lett. 58, 785 (2002).

[35] D. B. Lukatsky and S. A. Safran, Europhys. Lett. 60, 629 (2002).

[36] Private communication with a referee of this paper.

[37] Z.-S. Huang, Master thesis, Hong Kong University of Science and Technology, 2007; Available at http://lbxml.ust.hk/ th_imgo/b987318.pdf

[38] E. A. van Nierop, M. A. Stijnman, and S. Hilgenfeldt, Europhys. Lett. 72, 671 (2005).

[39] Y.-L. Han, A. Alsayed, M. Nobili, J. Zhang, T. C. Lubensky, and A. G. Yodh, Science 314, 626 (2006).

[40] A. San Paulo and R. Garcia, Phys. Rev. B 64, 193411 (2001).

[41] T. M. Fischer, P. Dhar, and P. Heinig, J. Fluid Mech. 558, 451 (2006).

[42] W. Chen and P. Tong, EPL 84, 28003 (2008).

[43] B. J. Park, J. P. Pantina, and E. M. Furst, Langmuir 24, 1686 (2008).

[44] See movies at http://physics.ust.hk/penger/prl.htm

[45] C. W. Helstrom, Probability and Stochastic Processes for Engineers, 2nd ed. (Macmillan Publishing Co., New York, 1991), p. 104.

[46] For a more general mathematical derivation, please refer to the webpage: http://mathworld.wolfram.com/ ChangeofVariablesTheorem.html and the references therein. 